\title{
The backward Monte Carlo method for semiconductor device simulation
}

\author{
Markus Kampl ${ }^{1}$ (D) Hans Kosina ${ }^{1}$
}

Published online: 2 August 2018

(c) The Author(s) 2018

\begin{abstract}
A backward Monte Carlo method for the numerical solution of the semiconductor Boltzmann equation is presented. The method is particularly suited to simulate rare events. The general theory of the backward Monte Carlo method is described, and several estimators for the contact current are derived from that theory. The transition probabilities for the construction of the backward trajectories are chosen so as to satisfy the principle of detailed balance. This property guarantees stability of the numerical method and allows for a clear physical interpretation of the estimators. A symmetric sampling method which generates wave vectors always in pairs symmetric to the origin can be shown to yield zero current exactly as thermal equilibrium is approached. The properties of the different estimators are evaluated by simulation of an $n$-channel MOSFET. Quantities varying over many orders of magnitude can be resolved with ease. Such quantities are the drain current in the sub-threshold region, the high-energy tail of the carrier distribution function, and the so-called acceleration integral which varies over 30 orders in the example shown.
\end{abstract}

Keywords Backward Monte Carlo method · Semiconductor Boltzmann equation · Device simulation · Electron distribution function $\cdot$ MOSFET $\cdot$ Rare events

\section{Introduction}

The Boltzmann Equation (BE) describes the motion of charge carriers in a semiconductor as semi-classical and can be used to model the electrical properties of semiconductor devices. The BE can be solved by means of the Monte Carlo method $[1,2]$ for realistic device structures and for realistic scattering and band structure models. The physically transparent and commonly used forward Monte Carlo (FMC) method, however, shows severe drawbacks in terms of computation time and statistical error when statistically rare events are to be simulated. A solution method that overcomes this drawback is the backward Monte Carlo (BMC) method. This method was introduced in the field of semi-classical transport at the end of the 1980s $[3,4]$. These early algorithms turned out to be numerically unstable, as the carrier energy tends to grow indefinitely on a trajectory that is followed back in time. A numerically stable algorithm was proposed in 2003 [5].

Markus Kampl

kampl@iue.tuwien.ac.at

1 Institute for Microelectronics, TU Wien, Gusshausstrasse 27-29, 1040 Vienna, Austria
Since the backward transition rates are chosen to obey the principle of detailed balance, a runaway of the carrier energy along a backward trajectory is avoided. From a practical point of view, this means that the scattering rates of the forward method can be used in the backward method as well [5].

The principle of the BMC method for the solution of a boundary value problem is to choose a set of states in phase space and trace trajectories from these states back in time until a contact is reached. The value of the given distribution function (DF) at the contact determines the statistical weight of the backward trajectory and consequently its contribution to the estimator of interest. To calculate a current which is controlled by an energy barrier, one would typically choose states at the top of the barrier and estimate the current from these states, see Fig. 1. If the barrier is high, a forward trajectory is very unlikely to reach the top of the barrier, whereas in the backward method only these unlikely states are considered, and no computation time is wasted with the vast majority of trajectories that do not overcome the barrier.

It is also possible to combine the backward and the forward MC method. Once a backward trajectory with an initial state $\left(\mathbf{k}_{0}, \mathbf{r}_{0}\right)$ is calculated and the statistical weight of that state is determined, a forward trajectory can be started from the 


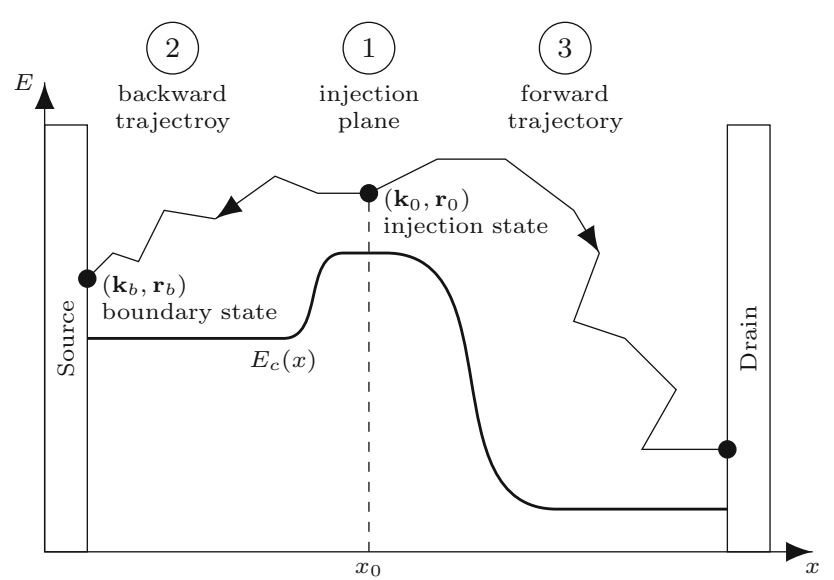

Fig. 1 Principle of the BFMC method for a MOSFET. The injected particle has a chosen state in $r$-and $k$-space. It is traced back in time to its origin to calculate the weight (probability) of itself. The observables are calculated from the weighted forward trajectory

very same state (Fig. 1). The mean values of interest are then calculated from a set of forward trajectories in the usual manner [6].

\section{Theory of the backward Monte Carlo method}

In semi-classical transport theory, carriers are treated as point-like particles. Their dynamic is determined by the equations of motion in phase space.

$\frac{\mathrm{d} \mathbf{k}}{\mathrm{d} t}=\mathbf{F}(\mathbf{r}, t), \quad \frac{\mathrm{d} \mathbf{r}}{\mathrm{d} t}=\mathbf{v}(\mathbf{k})$

The force field $\mathbf{F}$ takes into account electric and magnetic fields. If only the electric field $\mathbf{E}$ is present, the force field is given by $\mathbf{F}(\mathbf{r}, t)=(q / \hbar) \mathbf{E}(\mathbf{r}, t)$, where $q$ is the charge of the carrier. The carrier's group velocity $\mathbf{v}$ is related to the band energy $\mathcal{E}(\mathbf{k})$ by $\mathbf{v}=\hbar^{-1} \nabla \mathcal{E}(\mathbf{k})$. A phase space trajectory satisfying the initial conditions $\mathbf{K}_{0}\left(t_{0}\right)=\mathbf{k}_{0}$ and $\mathbf{R}_{0}\left(t_{0}\right)=\mathbf{r}_{0}$ can be obtained by formal integration of the equations of motion.

$$
\begin{aligned}
& \mathbf{K}_{0}(t)=\mathbf{k}_{0}+\int_{t_{0}}^{t} \mathbf{F}\left(\mathbf{R}_{0}(\tau), \tau\right) \mathrm{d} \tau \\
& \mathbf{R}_{0}(t)=\mathbf{r}_{0}+\int_{t_{0}}^{t} \mathbf{v}\left(\mathbf{K}_{0}(\tau)\right) \mathrm{d} \tau
\end{aligned}
$$

The BE describes the dynamics of an ensemble of semiclassical carriers in a semiconductor. The BE for the timeand position-dependent transport problem reads

$$
\begin{aligned}
& \left(\frac{\partial}{\partial t}+\mathbf{v}(\mathbf{k}) \cdot \nabla_{\mathbf{r}}+\mathbf{F}(\mathbf{r}, t) \cdot \nabla_{\mathbf{k}}\right) f(\mathbf{k}, \mathbf{r}, t) \\
& \quad=Q[f](\mathbf{k}, \mathbf{r}, t), \quad \mathbf{r} \in D
\end{aligned}
$$

This equation is posed in the simulation domain $D$ and has to be supplemented by boundary and initial conditions. The distribution function is commonly normalized as $\int_{D} \mathrm{~d}^{3} r \int \mathrm{d}^{3} k f(\mathbf{k}, \mathbf{r}, t)=4 \pi^{3} N_{D}(t)$, with $N_{D}$ denoting the number of carriers contained in the semiconductor domain $D$. The scattering operator $Q=Q_{\mathrm{g}}-Q_{1}$ consists of a gain and a loss term, respectively.

For the purpose of outlining the theory of the Backward MC method we neglect many-body effects for the moment. In this case, the scattering operator will be a linear integral operator.

$$
\begin{aligned}
Q_{\mathrm{g}}[f](\mathbf{k}, \mathbf{r}, t) & =\int f\left(\mathbf{k}^{\prime}, \mathbf{r}, t\right) S\left(\mathbf{k}^{\prime}, \mathbf{k}, \mathbf{r}\right) \mathrm{d}^{3} k^{\prime} \\
Q_{1}[f](\mathbf{k}, \mathbf{r}, t) & =\lambda(\mathbf{k}, \mathbf{r}) f(\mathbf{k}, \mathbf{r}, t)
\end{aligned}
$$

Here $S\left(\mathbf{k}^{\prime}, \mathbf{k}, \mathbf{r}\right) \mathrm{d}^{3} k$ denotes the scattering rate from a state $\mathbf{k}^{\prime}$ to states in $\mathrm{d}^{3} k$ around $\mathbf{k}$, and $\lambda(\mathbf{k}, \mathbf{r})=\int S\left(\mathbf{k}, \mathbf{k}^{\prime}, \mathbf{r}\right) \mathrm{d}^{3} k^{\prime}$ is the total scattering rate. Some scattering mechanisms such as ionized impurity scattering are position-dependent. In the following, however, explicit writing of the r-dependence of the scattering rate is omitted for the sake of brevity.

If many-body effects such as carrier-carrier scattering and degeneracy are to be considered, one has to resort to the established approximations [7].

\subsection{Integral form of the Boltzmann equation}

The BE (4) can be formally integrated over a phase space trajectory. Details can be found in [8].

$$
\begin{aligned}
& f\left(\mathbf{k}_{0}, \mathbf{r}_{0}, t_{0}\right) \\
& \quad=\int_{0}^{t_{0}} \mathrm{~d} t_{1} \int \mathrm{d}^{3} k_{1} K\left(\mathbf{k}_{0}, t_{0}, \mathbf{k}_{1}, t_{1}\right) f\left(\mathbf{k}_{1}, \mathbf{R}\left(t_{1}\right), t_{1}\right) \\
& \quad+f_{0}\left(\mathbf{k}_{0}, \mathbf{r}_{0}, t_{0}\right)
\end{aligned}
$$

The resulting integral equation (7) represents the generalization of Chamber's path integral [9]. The source term $f_{0}$ contains the initial distribution in case of an initial value problem [10], or the boundary distribution in case of a boundary value problem [8]. The integral kernel is of the form

$K\left(\mathbf{k}_{0}, t_{0}, \mathbf{k}_{1}, t_{1}\right)=S\left(\mathbf{k}_{1}, \mathbf{K}_{0}\left(t_{1}\right)\right) \exp \left(-\int_{t_{1}}^{t_{0}} \lambda\left(\mathbf{K}_{0}(\tau)\right) \mathrm{d} \tau\right)$

The trajectory $\mathbf{K}_{0}(\tau)$ passes through $\mathbf{k}_{0}$ at time $t_{0}$. From a physical point of view, the kernel (8) describes a transition from $\left(\mathbf{k}_{1}, t_{1}\right)$ to $\left(\mathbf{k}_{0}, t_{0}\right)$. 


\subsection{Probability density functions}

The components of the kernel (8) are used to construct probability density functions (PDF). From the scattering rate $S$, one can define a PDF of the after-scattering states $\mathbf{k}_{a}$.

$p_{k}\left(\mathbf{k}_{a} \mid \mathbf{k}_{b}\right)=\frac{S\left(\mathbf{k}_{b}, \mathbf{k}_{a}\right)}{\lambda\left(\mathbf{k}_{b}\right)}$

The total scattering rate $\lambda\left(\mathbf{k}_{b}\right)=\int S\left(\mathbf{k}_{b}, \mathbf{k}_{a}\right) \mathrm{d}^{3} k_{a}$ serves as a normalization factor. Conversely, the PDF of the beforescattering states $\mathbf{k}_{b}$ is defined as

$p_{k}^{*}\left(\mathbf{k}_{b} \mid \mathbf{k}_{a}\right)=\frac{S\left(\mathbf{k}_{b}, \mathbf{k}_{a}\right)}{\lambda^{*}\left(\mathbf{k}_{a}\right)}$.

The normalization factor is given by the backward scattering rate, $\lambda^{*}\left(\mathbf{k}_{a}\right)=\int S\left(\mathbf{k}_{b}, \mathbf{k}_{a}\right) \mathrm{d}^{3} k_{b}$.

The path integral in (8) is related to the PDF of the backward free-flight time $t_{1}$. The PDF is denoted by $p_{t}^{*}$.

$$
\begin{aligned}
& p_{t}^{*}\left(t_{1} \mid t_{0} ; \mathbf{k}_{0}\right) \\
& \quad=\lambda\left(\mathbf{K}_{0}\left(t_{1}\right)\right) \exp \left(-\int_{t_{1}}^{t_{0}} \lambda\left(\mathbf{K}_{0}(\tau)\right) \mathrm{d} \tau\right), \quad t_{1}<t_{0}
\end{aligned}
$$

The PDF is normalized as follows:

$$
\int_{-\infty}^{t_{0}} p_{t}^{*}\left(t_{1} \mid t_{0}\right) \mathrm{d} t_{1}=1
$$

It can be easily shown that the equations of motion (1) are form-invariant under the transformation $t^{*}=-t, \mathbf{k}^{*}=-\mathbf{k}$, and $\mathbf{v}^{*}=-\mathbf{v}$ :

$\frac{\mathrm{d} \mathbf{k}^{*}}{\mathrm{~d} t^{*}}=\mathbf{F}(\mathbf{r}, t), \quad \frac{\mathrm{d} \mathbf{r}}{\mathrm{d} t^{*}}=\mathbf{v}^{*}$

Increasing $t^{*}$ means a further progression into the past. Note that the position vector $\mathbf{r}$ and the force field $\mathbf{F}$ need not be inverted. Under the transformation $\tau^{*}=-\tau$, the integration boundaries in (11) get interchanged, and the PDF takes on the form of a forward flight-time distribution.

$$
\begin{aligned}
& p_{t}\left(t_{1}^{*} \mid t_{0}^{*} ; \mathbf{k}_{0}^{*}\right) \\
& =\lambda\left(\mathbf{K}_{0}^{*}\left(t_{1}^{*}\right)\right) \exp \left(-\int_{t_{0}^{*}}^{t_{1}^{*}} \lambda\left(\mathbf{K}_{0}^{*}\left(\tau^{*}\right)\right) \mathrm{d} \tau^{*}\right), \quad t_{1}^{*}>t_{0}^{*}
\end{aligned}
$$

The PDF satisfies the normalization

$$
\int_{t_{0}^{*}}^{\infty} p_{t}\left(t_{1}^{*} \mid t_{0}^{*}\right) \mathrm{d} t_{1}^{*}=1 .
$$

\subsection{The backward MC method}

The integral equation can be solved by means of the Markovchain Monte Carlo method [3,4]. Starting from (7) will result in a backward Monte Carlo method, which means that the time instants $t_{i}$, when in the simulation scattering events occur, form a descending sequence: $t_{0}>t_{1}>t_{2}>\cdots$.

Conversely, the more familiar forward Monte Carlo method is based on the adjoint equation of (7), see [8]. In the course of the simulation, an ascending sequence of time instants, $t_{0}<t_{1}<t_{2}<\cdots$, will be generated.

In the BMC method, the distribution function $f$ in a given phase space point $\mathbf{k}_{0}, \mathbf{r}_{0}$ is estimated by the following sample mean:

$f\left(\mathbf{k}_{0}, \mathbf{r}_{0}, t_{0}\right) \simeq \frac{1}{N} \sum_{s=1}^{N} \mu_{s}^{(n(s))}\left(\mathbf{k}_{0}, \mathbf{r}_{0}, t_{0}\right)$

here $N$ denotes the number of trajectories, and $n(s)$ is the order of the $s$-th numerical trajectory, which is the number of scattering events occurring in the interval $\left[0, t_{0}\right]$. In this work, we discuss two specific choices of the estimator $\mu^{(n)}$.

\subsection{Transition rate derived from mathematical considerations}

In the original works [3] and [4], $S\left(\mathbf{k}_{b}, \mathbf{k}_{a}\right)$ is interpreted as the unnormalized distribution of the before-scattering states $\mathbf{k}_{b}$, and consequently the normalized PDF (10) is employed. Using the transition density,

$P\left(\mathbf{k}_{1}, t_{1} \mid \mathbf{k}_{0}, t_{0}\right)=p_{k}^{*}\left(\mathbf{k}_{1} \mid \mathbf{K}_{0}\left(t_{1}\right)\right) p_{t}\left(t_{1} \mid t_{0} ; \mathbf{k}\right)$

the estimator in (16) becomes

$$
\begin{aligned}
& \mu^{(n)}\left(\mathbf{k}_{0}, \mathbf{r}_{0}, t_{0}\right) \\
& \quad=\frac{\lambda^{*}\left(\mathbf{K}_{0}\left(t_{1}\right)\right)}{\lambda\left(\mathbf{K}_{0}\left(t_{1}\right)\right)} \ldots \frac{\lambda^{*}\left(\mathbf{K}_{n-1}\left(t_{n}\right)\right)}{\lambda\left(\mathbf{K}_{n-1}\left(t_{n}\right)\right)} f_{\text {in }}\left(\mathbf{K}_{n}(0), \mathbf{R}_{n}(0)\right) .
\end{aligned}
$$

Here $f_{\text {in }}$ denotes the initial distribution. The sketch of a kspace trajectory of second order $(n=2)$ is shown in Fig. 2

Although the MC algorithm based on the estimator (18) is consistently derived from the integral form of the BE, computer experiments reveal a stability problem. The particle energy becomes very high when the trajectory is followed backward in time. The initial distribution takes on very small values at high energies, so that many realizations of the estimator will be very small. With small probability, the particle energy will stay low, where the initial distribution is large. These rare events give large contributions to the estimator, resulting in a large variance. The computer experiments show that the variance increases rapidly with time. However, for a given time $t$ the variance of the estimator is finite. 


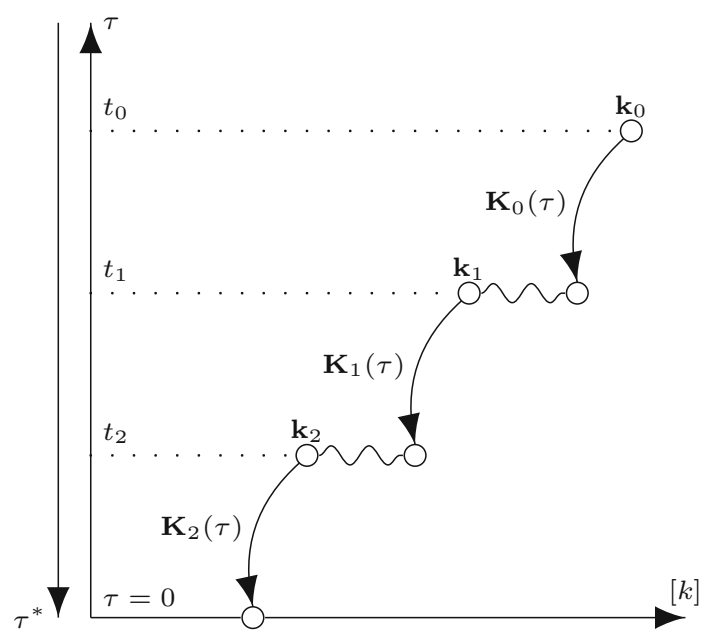

Fig. 2 Sketch of a backward trajectory starting at time $t$ and reaching time 0 after three free flights. The symbols used in (18) are shown

\subsection{Transition rate derived from physical considerations}

The time evolution of the particle energy can be understood from a property of the scattering rate known as the principle of detailed balance. This property ensures that in any system particles scatter preferably to lower energies. If the backward transition rate (10) is employed for trajectory construction, in the simulation the principle of detailed balance is inverted, and scattering to higher energies is preferred.

The principle of detailed balance is reflected by the following symmetry property of the scattering rate:

$S\left(\mathbf{k}_{i}, \mathbf{k}_{j}\right)=S\left(\mathbf{k}_{j}, \mathbf{k}_{i}\right) \mathrm{e}^{\beta_{D}\left(\mathcal{E}\left(\mathbf{k}_{i}\right)-\mathcal{E}\left(\mathbf{k}_{j}\right)\right)}$,

where $\beta_{D}=\left(k_{B} T_{\mathrm{D}}\right)^{-1}$ with $T_{\mathrm{D}}$ being the device temperature, and $\mathcal{E}(\mathbf{k})$ denoting the carrier energy. The stability problem can be solved by using the forward scattering rate also for the construction of the backward trajectory and changing the estimator accordingly. In the transition density, the forward PDF (9) is employed.

$P\left(\mathbf{k}_{1}, t_{1} \mid \mathbf{k}_{0}, t_{0}\right)=p_{k}\left(\mathbf{k}_{1} \mid \mathbf{K}_{0}\left(t_{1}\right)\right) p_{t}\left(t_{1} \mid t_{0} ; \mathbf{k}_{0}\right)$

The estimator in (16) becomes

$\mu^{(n)}(\mathbf{k}, \mathbf{r}, t)=\mathrm{e}^{\beta_{D} \Delta \mathcal{E}_{1}} \ldots \mathrm{e}^{\beta_{D} \Delta \mathcal{E}_{n}} f_{\text {in }}\left(\mathbf{K}_{n}(0), \mathbf{R}_{n}(0)\right)$.

Here the difference in carrier energy induced by the $l$-th scattering event is denoted by $\Delta \mathcal{E}_{l}$.

The result obtained for the initial value problem can be reformulated straightforward for the stationary boundary value problem. We define the total electron energy as

$H(\mathbf{k}, \mathbf{r})=\mathcal{E}(\mathbf{k})+E_{C}(\mathbf{r})$ where $E_{C}(\mathbf{r})$ is the conduction band edge. In the following, we shall consistently label the starting point of a backward trajectory as $\left(\mathbf{k}_{0}, \mathbf{r}_{0}\right)$, and the endpoint at a Dirichlet boundary as $\left(\mathbf{k}_{b}, \mathbf{r}_{b}\right)$, see Fig. 1. A Dirichlet boundary is imposed by an ohmic contact, where an equilibrium distribution can be assumed. The boundary distribution at a contact will be referred to as $f_{b}$. Inelastic scattering events cause a difference in the total energy along a trajectory. Using the energy balance equation

$H\left(\mathbf{k}_{b}, \mathbf{r}_{b}\right)-H\left(\mathbf{k}_{0}, \mathbf{r}_{0}\right)=\sum_{l} \Delta \mathcal{E}_{l}$

the estimator (21) for the distribution function at a given point $\left(\mathbf{k}_{0}, \mathbf{r}_{0}\right)$ becomes

$$
f\left(\mathbf{k}_{0}, \mathbf{r}_{0}\right)=\frac{1}{M} \sum_{i=1}^{M} f_{b}\left(\mathbf{k}_{b, i}, \mathbf{r}_{b . i}\right) \mathrm{e}^{\beta_{D}\left(H\left(\mathbf{k}_{b, i}, \mathbf{r}_{b, i}\right)-H\left(\mathbf{k}_{0}, \mathbf{r}_{0}\right)\right)} .
$$

Here $M$ is the number of backward trajectories started from the point $\left(\mathbf{k}_{0}, \mathbf{r}_{0}\right)$. Note that the backward trajectory is constructed in the very same manner as a forward trajectory. Using the forward PDF (14) to generate the free-flight time means that we have inverted the time axis and are progressing along the negative time axis. The selection of the scattering mechanism and the calculation of the after-scattering state are also identical to the forward algorithm.

\section{Current estimators}

We start with the general definition of current density,

$\mathbf{J}=\frac{2}{(2 \pi)^{3}} q \int_{\text {BZ }} \mathbf{v}(\mathbf{k}) f(\mathbf{k}, \mathbf{r}) \mathrm{d}^{3} k$,

where $f(\mathbf{k}, \mathbf{r})$ is the unknown solution of the BE. The current through an area of size $y_{\mathrm{m}} \times W$ in the $y z$-plane located at $x=x_{0}$ is obtained by integration.

$I=\int_{0}^{y_{\mathrm{m}}} \int_{z=0}^{W} \mathbf{J} \cdot \mathrm{d} \mathbf{A}$

Substituting the surface element $\mathrm{d} \mathbf{A}=\mathbf{e}_{x} W \mathrm{~d} y_{0}$ and the current density (25), and assuming the distribution function to be uniform in the $z$-direction, the current becomes

$I=\frac{q}{4 \pi^{3}} W \int_{0}^{y_{\mathrm{m}}} \int_{\mathrm{BZ}} v_{x}\left(\mathbf{k}_{0}\right) f\left(\mathbf{k}_{0}, x_{0}, y_{0}\right) \mathrm{d}^{3} k_{0} \mathrm{~d} y_{0}$, 
where $W$ is the device width. In principle, the integral in (27) could be evaluated by numerical integration, whereby the values of the distribution function at the discrete points $\left(\mathbf{k}_{0}, \mathbf{r}_{0}\right)$ are estimated by (24). However, it is more convenient to employ Monte Carlo integration instead. For this purpose, the current has to be expressed as an expectation value. This is accomplished by introducing a PDF $p_{0}\left(\mathbf{k}_{0}, y_{0}\right)$ which can be chosen freely, and reformulating (27) as:

$I=q W \int_{0}^{y_{\mathrm{m}}} \int_{\mathrm{BZ}} \mu\left(\mathbf{k}_{0}, y_{0} ; x_{0}\right) p_{0}\left(\mathbf{k}_{0}, y_{0}\right) \mathrm{d}^{3} k_{0} \mathrm{~d} y_{0} \equiv q W E\{\mu\}$

with

$\mu\left(\mathbf{k}_{0}, y_{0} ; x_{0}\right)=\frac{v_{x}\left(\mathbf{k}_{0}\right) f\left(\mathbf{k}_{0}, x_{0}, y_{0}\right)}{4 \pi^{3} p_{0}\left(\mathbf{k}_{0}, y_{0}\right)}$

In a Monte Carlo simulation, the expectation value is estimated by a sample mean [11].

$I=q W E\{\mu\} \approx q W \frac{1}{N} \sum_{i=1}^{N} \mu\left(\mathbf{k}_{0, i}, y_{0, i} ; x_{0}\right)$

Here $N$ is the number of sampling points. In the next step, the unknown distribution function $f$ in (28) is expressed through the estimator (24). This would in general convert (29) into a double sum of $N \times M$ elements. However, it is sufficient to start only one backward trajectory from each sampling point $\left(\mathbf{k}_{0, i}, y_{0, i}\right)$, so that one can set $M=1$ in (24). With this assumption the estimator $\mu$ in (28) becomes:

$\mu\left(\mathbf{k}_{0}, y_{0} ; x_{0}\right)=\frac{v_{x}\left(\mathbf{k}_{0}\right) f_{b}\left(\mathbf{k}_{b}, \mathbf{r}_{b}\right)}{4 \pi^{3} p_{0}\left(\mathbf{k}_{0}, y_{0}\right)} \mathrm{e}^{\beta_{D}\left(H\left(\mathbf{k}_{b}, \mathbf{r}_{b}\right)-H\left(\mathbf{k}_{0}, \mathbf{r}_{0}\right)\right)}$

This is the basic equation from which various current estimators can be derived. In the following sections, we will discuss different choices of the injection PDF $p_{0}$ and the properties of the resulting current estimators. In (30), $\mathbf{k}_{0}$ and $y_{0}$ are random variables, whereas $x_{0}$ is a given parameter. In the following, $x_{0}$ is often omitted from the argument list for the sake of brevity.

\subsection{The boundary distribution}

The distribution function is close to thermal equilibrium at ohmic contacts. Therefore, suitable choices for the boundary distribution $f_{b}$ are a Maxwell-Boltzmann or a Fermi-Dirac distribution. Throughout this work, we assume an equilibrium Boltzmann distribution:

$f_{b}(\mathbf{k}, \mathbf{r})=C(\mathbf{r}) \mathrm{e}^{-\beta_{\mathrm{D}} \mathcal{E}(\mathbf{k})}$
Two normalization integrals will be needed in the following. The first one is the partition function $Z(T)$ defined as [12]:

$Z(T)=\int_{B Z} \mathrm{e}^{-\beta(T) \mathcal{E}(\mathbf{k})} \mathrm{d}^{3} k$

The second integral is defined as

$V(T)=\int_{\text {BZ }}\left|v_{x}\left(\mathbf{k}_{0}\right)\right| \mathrm{e}^{-\beta(T) \mathcal{E}\left(\mathbf{k}_{0}\right)} \mathrm{d}^{3} k$.

The integrations over the Brillouin zone (BZ) are carried out numerically. From these two quantities, the injection velocity is obtained:

$v_{\text {inj }}(T)=\frac{V(T)}{Z(T)}$

The definition of the electron concentration

$n(\mathbf{r})=\frac{1}{4 \pi^{3}} \int_{\text {BZ }} f_{b}(\mathbf{k}, \mathbf{r}) \mathrm{d}^{3} k$

is used to determine the normalization constant $C$ in (31).

$C(\mathbf{r})=\frac{4 \pi^{3} n(\mathbf{r})}{Z\left(T_{\mathrm{D}}\right)}$

\subsection{Injection from an equilibrium Maxwellian}

The PDF $p_{0}$ from which the starting points of the backward trajectories are generated can be expressed as a product of two independent PDFs:

$p_{0}\left(\mathbf{k}_{0}, y_{0}\right)=f_{0}\left(\mathbf{k}_{0}\right) p_{y}\left(y_{0}\right)$

The PDF of the injection coordinate $y_{0}$ is assumed to be proportional to the electron concentration.

$p_{y}\left(y_{0}\right)=\frac{n\left(x_{0}, y_{0}\right)}{\int_{0}^{y_{\mathrm{m}}} n\left(x_{0}, y\right) \mathrm{d} y}$

The first choice of the injection distribution $f_{0}$ we consider is a normalized Boltzmann distribution at device temperature $T_{\mathrm{D}}$.

$f_{0}\left(\mathbf{k}_{0}\right)=\frac{1}{Z\left(T_{\mathrm{D}}\right)} \mathrm{e}^{-\beta_{D} \mathcal{E}\left(\mathbf{k}_{0}\right)}$

Inserting the boundary distribution (31) and the injection distribution (39) in (30) gives the following current estimator:

$\mu=v_{x}\left(\mathbf{k}_{0}\right) \frac{\mathrm{e}^{\beta_{\mathrm{D}}\left(E_{\mathrm{C}}\left(\mathbf{r}_{\mathrm{b}}\right)-E_{\mathrm{C}}\left(\mathbf{r}_{0}\right)\right)}}{p_{y}\left(y_{0}\right)} n\left(\mathbf{r}_{\mathrm{b}}\right)$ 
Note that both the Boltzmann factors $\mathrm{e}^{-\beta_{D} \mathcal{E}\left(\mathbf{k}_{0}\right)}$ and $\mathrm{e}^{-\beta_{D} \mathcal{E}\left(\mathbf{k}_{b}\right)}$ are canceled out of this expression. To generate wave vectors from the equilibrium distribution (39), one can perform a single-particle simulation at zero electric field and sample the trajectory at equidistant time steps.

\subsection{Injection from a velocity-weighted Maxwellian}

The second choice for $f_{0}$ we consider is a velocity-weighted Maxwellian at equilibrium temperature $T_{\mathrm{D}}$.

$f_{0}\left(\mathbf{k}_{0}\right)=\frac{1}{V\left(T_{\mathrm{D}}\right)}\left|v_{x}\left(\mathbf{k}_{0}\right)\right| \mathrm{e}^{-\beta_{D} \mathcal{E}\left(\mathbf{k}_{0}\right)}$

This choice is motivated by the fact that in (30) a term $v_{x}\left(k_{0}\right) \mathrm{e}^{-\beta_{D} \mathcal{E}\left(k_{0}\right)}$ occurs in the numerator. Division by (41) will essentially cancel out this term. This reduces the $k_{0^{-}}$ dependence of the estimator which is expected to reduce its variance. Inserting the boundary distribution (31) and the injection distribution (41) in (30) yields

$\mu=\operatorname{sign}\left(v_{x}\left(\mathbf{k}_{0}\right)\right) v_{\text {inj }}\left(T_{\mathrm{D}}\right) \frac{\mathrm{e}^{\beta_{\mathrm{D}}\left(E_{\mathrm{C}}\left(\mathbf{r}_{b}\right)-E_{\mathrm{C}}\left(\mathbf{r}_{0}\right)\right)}}{p_{y}\left(y_{0}\right)} n\left(\mathbf{r}_{b}\right)$.

In this equation, $\operatorname{sign}\left(v_{x}\right)$ denotes the sign of the velocity component $v_{x}$, and $v_{\text {inj }}$ is the injection velocity defined by (34).

\subsection{Injection from a non-equilibrium Maxwellian}

For some applications, it can be useful to generate the initial points $\mathbf{k}_{0}$ from a Maxwellian at a temperature $T_{0}$ different from the device temperature $T_{\mathrm{D}}$. Especially when calculating quantities depending on the high-energy tail of the distribution, an injection temperature $T_{0}>T_{\mathrm{D}}$ will be beneficial as it enhances the number of initial points at higher energies. We consider a non-equilibrium Maxwellian of the form

$f_{0}\left(\mathbf{k}_{0}\right)=\frac{1}{Z\left(T_{0}\right)} \mathrm{e}^{-\beta_{0} \mathcal{E}\left(\mathbf{k}_{0}\right)}$

Inserting this injection distribution in (30) gives an estimator which will be denoted by $\eta$ :

$$
\begin{aligned}
\eta\left(\mathbf{k}_{0}, y_{0}\right)= & \frac{Z\left(T_{0}\right)}{Z\left(T_{\mathrm{D}}\right)} v_{x}\left(\mathbf{k}_{0}\right) \frac{\mathrm{e}^{\beta_{D}\left(E_{\mathrm{C}}\left(\mathbf{r}_{b}\right)-E_{\mathrm{C}}\left(\mathbf{r}_{0}\right)\right)}}{p_{y}\left(y_{0}\right)} \\
& \times \mathrm{e}^{\left(\beta_{0}-\beta_{\mathrm{D}}\right) \mathcal{E}\left(\mathbf{k}_{0}\right)} n\left(\mathbf{r}_{b}\right)
\end{aligned}
$$

It will be shown below that the sample mean of $\eta$,

$$
I=q W \frac{1}{N} \sum_{i=1}^{N} \eta\left(\mathbf{k}_{0, i}, y_{0, i}\right)
$$

can be reformulated as a weighted average of the form:

$I=q W \frac{\sum_{i=1}^{N} \mu\left(\mathbf{k}_{0, i}, y_{0, i}\right) w\left(\mathbf{k}_{0, i}\right)}{\sum_{i=1}^{N} w\left(\mathbf{k}_{0, i}\right)}$

Here $\mu$ is given by (40) and the weight factor $w$ is defined as

$w\left(k_{0}\right)=\mathrm{e}^{\left(\beta_{0}-\beta_{D}\right) \mathcal{E}\left(\mathbf{k}_{0}\right)}$.

The derivation of (46) is not obvious. With (40) and (47), expression (44) can be rewritten as

$\eta\left(\mathbf{k}_{0}, y_{0}\right)=\frac{Z\left(T_{0}\right)}{Z\left(T_{\mathrm{D}}\right)} \mu\left(\mathbf{k}_{0}, y_{0}\right) w\left(\mathbf{k}_{0}\right)$

A drawback of this formulation is that the normalization factor $Z\left(T_{0}\right)$ has to be recalculated for each $T_{0}$. The numerical integration over the Brillouin zone, however, can be avoided by a proper application of Monte Carlo integration. We express the integral in (32) as an expectation value which in turn will be estimated by a sample mean.

$Z\left(T_{\mathrm{D}}\right)=\int_{\mathrm{BZ}} \frac{\mathrm{e}^{-\beta_{D} \mathcal{E}\left(\mathbf{k}_{0}\right)}}{f_{0}\left(\mathbf{k}_{0}\right)} f_{0}\left(\mathbf{k}_{0}\right) \mathrm{d}^{3} k_{0}$

Inserting (43) yields

$Z\left(T_{\mathrm{D}}\right)=Z\left(T_{0}\right) \int_{\mathrm{BZ}} w\left(k_{0}\right) f_{0}\left(\mathbf{k}_{0}\right) \mathrm{d}^{3} k_{0} \equiv Z\left(T_{0}\right) E\{w\}$

From this equation, we obtain the ratio of the normalization factors as

$\frac{Z\left(T_{\mathrm{D}}\right)}{Z\left(T_{0}\right)}=E\{w\} \approx \frac{1}{N} \sum_{i=1}^{N} w\left(\mathbf{k}_{0, i}\right)$

Substituting this ratio in (48) represents the final step in the reformulation of the sample mean to arrive at (46).

In the case of a velocity-weighted Maxwellian with $T_{0} \neq$ $T_{\mathrm{D}}$,

$f_{0}\left(\mathbf{k}_{0}\right)=\frac{1}{V\left(T_{0}\right)}\left|v_{x}\left(\mathbf{k}_{0}\right)\right| \mathrm{e}^{-\beta_{0} \mathcal{E}\left(\mathbf{k}_{0}\right)}$

a similar procedure can be applied. In the weighted average (46) the estimator (42) and the very same weight (47) have to be used.

\subsection{Injection from the equilibrium concentration}

All estimators discussed so far depend on the injection coordinate $y_{0}$ through the term $\mathrm{e}^{\beta_{D} E_{\mathrm{C}}\left(\mathbf{r}_{0}\right)} / p_{y}\left(y_{0}\right)$. However, this 
dependence is weak and can even be eliminated from the estimator by choosing the injection distribution as

$p_{0}\left(\mathbf{k}_{0}, y_{0} ; x_{0}\right)=\frac{1}{A\left(T_{\mathrm{D}}\right)}\left|v_{x}\left(\mathbf{k}_{0}\right)\right| \mathrm{e}^{-\beta_{D} H\left(\mathbf{k}_{0}, x_{0}, y_{0}\right)}$

Inserting (22) in (52) again yields a product of two independent PDFs.

$p_{0}\left(\mathbf{k}_{0}, y_{0}\right)=f_{0}\left(\mathbf{k}_{0}\right) \tilde{p}_{y}\left(y_{0}\right)$

Here $f_{0}$ is given by (41), and $\tilde{p}_{y}$ is defined as

$\tilde{p}_{y}\left(y_{0}\right)=\frac{\tilde{n}\left(x_{0}, y_{0}\right)}{B\left(T_{\mathrm{D}}\right)}$

with $\tilde{n}\left(x_{0}, y_{0}\right)=\mathrm{e}^{-\beta_{D} E_{C}\left(x_{0}, y_{0}\right)}$. This quantity is up to a constant, the equilibrium concentration, determined by the band edge energy $E_{C}$. On the other hand, $n$ in (38) represents the actual carrier concentration as obtained from a device simulation. With the normalization integral in (54) defined as

$B\left(T_{\mathrm{D}}\right)=\int_{0}^{y_{\mathrm{m}}} \tilde{n}\left(x_{0}, y\right) \mathrm{d} y$,

the total normalization factor $A$ in (52) becomes

$A\left(T_{\mathrm{D}}\right)=V\left(T_{\mathrm{D}}\right) B\left(T_{\mathrm{D}}\right)$.

Using the boundary distribution (31) and the injection distribution (52), the current estimator (30) can be reformulated as:

$\mu=\operatorname{sign}\left(v_{x}\left(\mathbf{k}_{0}\right)\right) v_{\text {inj }}\left(T_{\mathrm{D}}\right) B\left(T_{\mathrm{D}}\right) n\left(\mathbf{r}_{b}\right) \mathrm{e}^{\beta_{D} E_{C}\left(\mathbf{r}_{b}\right)}$

Other than the estimators discussed above, this estimator is independent of the injection coordinate $y_{0}$. A more transparent physical interpretation is achieved by expressing the equilibrium concentration $n\left(\mathbf{r}_{b}\right)$ as a function of the local quasi-Fermi level $F_{n}$.

$n\left(\mathbf{r}_{b}\right)=N_{C}\left(T_{\mathrm{D}}\right) \mathrm{e}^{\beta_{D}\left(F_{n}\left(\mathbf{r}_{b}\right)-E_{C}\left(\mathbf{r}_{b}\right)\right.}$

Here the effective density of states $N_{C}$ is related to the partition function $Z$ by $N_{C}=Z /\left(4 \pi^{3}\right)$. Also, the normalization factor $B$ will be expressed through an energy $\bar{E}_{C}$ defined as

$\bar{E}_{C}=-k_{B} T_{\mathrm{D}} \ln \left(\frac{B}{y_{\mathrm{m}}}\right)$.

$\bar{E}_{C}$ has the meaning of an average of the band edge energy over the injection coordinate $y_{0}$ :

$\mathrm{e}^{-\beta_{D} \bar{E}_{C}\left(x_{0}\right)}=\frac{1}{y_{\mathrm{m}}} \int_{0}^{y_{\mathrm{m}}} \mathrm{e}^{-\beta_{D} E_{C}\left(x_{0}, y_{0}\right)} \mathrm{d} y_{0}$
Expressing the estimator (57) in terms of the parameters $F_{n}$ and $\bar{E}_{C}$ gives

$\mu=y_{\mathrm{m}} N_{C}\left(T_{\mathrm{D}}\right) v_{\text {inj }}\left(T_{\mathrm{D}}\right) \operatorname{sign}\left(v_{x}\left(\mathbf{k}_{0}\right)\right) \mathrm{e}^{\beta_{D}\left(F_{n}\left(\mathbf{r}_{b}\right)-\bar{E}_{C}\right)}$.

This equation states that a backward trajectory represents an elementary particle flux $N_{C} v_{\text {inj }}$. This flux is multiplied by a statistical weight given by the e-function. The higher the energy of the starting point $\left(\bar{E}_{C}\right)$ with respect to the Fermi level at the trajectory end point $\left(F_{n}\right)$, the lower is the statistical weight. If a constant $\Delta \mathcal{E}$ were added to $\bar{E}_{C}$, the estimator $\mu$ and subsequently also the current $I$ would be scaled by the factor $\mathrm{e}^{-\beta_{D} \Delta \mathcal{E}}$. In other words, increasing the barrier height by some energy increment will result in an exponential decrease in current. This means that the exponential dependence of the thermionic current on the barrier height can be directly deduced from the current estimator (61).

\subsection{Symmetric sampling}

In thermodynamic equilibrium, the current will vanish due to the symmetry of the distribution function. Estimating the current in equilibrium by the BMC method will result in positive values of the estimator for nearly half of the backward trajectories, and in negative values for the other half. In the sample mean, these positive and negative values will cancel to a large extent, and the current will be nearly zero. However, the current will not be exactly zero because we have to work with finite sample sizes. This type of statistical error, however, can be easily eliminated by always generating positive and negative values of the estimator in pairs. When starting a backward trajectory from a state $\left(\mathbf{k}_{0}, y_{0}\right)$, we also start another one from the opposite momentum state $\left(-\mathbf{k}_{0}, y_{0}\right)$. As we will show below, this procedure will give $I=0$ exactly in thermal equilibrium without statistical error. One can also expect that this procedure will reduce the statistical error in situations close to thermal equilibrium.

Every estimator described above can be used to define a new estimator by taking the algebraic mean value:

$\mu_{\text {symm }}\left(\mathbf{k}_{0}, y_{0}\right)=\frac{\mu\left(\mathbf{k}_{0}, y_{0}\right)+\mu\left(-\mathbf{k}_{0}, y_{0}\right)}{2}$

Using (61) the new estimator will be of the form:

$$
\begin{aligned}
\mu_{\text {symm }}= & \frac{y_{\mathrm{m}} N_{C}\left(T_{\mathrm{D}}\right) v_{\text {inj }}\left(T_{\mathrm{D}}\right)}{2} \\
& \left(\mathrm{e}^{\beta_{D} F_{n}^{+}\left(\mathbf{r}_{b}\right)}-\mathrm{e}^{\beta_{D} F_{n}^{-}\left(\mathbf{r}_{b}\right)}\right) \mathrm{e}^{-\beta_{D} \bar{E}_{C}}
\end{aligned}
$$

In the following, we refer to the trajectory with $v_{x}\left(\mathbf{k}_{0}\right)>0$ as the plus-trajectory, and to that with $v_{x}\left(\mathbf{k}_{0}\right)<0$ as the minustrajectory. In (63), $F_{n}^{+}$denotes the quasi-Fermi level of the contact where the plus-trajectory has terminated, whereas 
$F_{n}^{-}$is the quasi-Fermi level of the contact where the minustrajectory has terminated.

In thermal equilibrium, the Fermi level $E_{\mathrm{F}}$ is constant throughout the device. Regardless of at which contact a backward trajectory ends, the Fermi level encountered will always be $F_{n}=E_{\mathrm{F}}$. The estimator (63) will identically vanish, and so will the current.

\subsection{Estimation of the statistical error}

Since all backward trajectories are statistically independent, one can easily find expressions for the statistical error of the simulation result.

We begin with the case that the injection states $\mathbf{k}_{0}$ are generated from an equilibrium distribution. In a Monte Carlo simulation, a sample $\left\{\mu_{1}, \mu_{2}, \ldots, \mu_{N}\right\}$ from a random variable $\mu$ is generated. Here, $\mu$ is a function of the random variables $\left(\mathbf{k}_{0}, y_{0}\right)$. Several such functions $\mu\left(\mathbf{k}_{0}, y_{0}\right)$ have been described in the preceding sections. The sample mean $\bar{\mu}$

$\bar{\mu}=\frac{1}{N} \sum_{i=1}^{N} \mu_{i}$

gives the current, $I=q W \bar{\mu}$, whereas the sample variance $s_{\mu}^{2}$ allows an estimate of the current's statistical error.

$s_{\mu}^{2}=\frac{1}{N-1}\left(\sum_{i=1}^{N} \mu_{i}^{2}-N \bar{\mu}^{2}\right)$

The standard deviation of the current is estimated as

$s_{I}=q W \frac{s_{\mu}}{\sqrt{N}} \Rightarrow \frac{s_{I}}{I}=\frac{1}{\sqrt{N}} \frac{s_{\mu}}{\bar{\mu}}$.

The relative standard deviation $s_{I} / I$ will be used as a measure for the statistical error in the following.

If the injection states are generated from a non-equilibrium distribution, we must consider the random variable $w$ defined by (47) and the random variable $\xi$ defined by

$\xi=\mu w$.

In the course of a Monte Carlo simulation, the sample means $\bar{\xi}$ and $\bar{w}$ have to be calculated in order to obtain the current:

$\bar{\xi}=\frac{1}{N} \sum_{i=1}^{N} \xi_{i}, \quad \bar{w}=\frac{1}{N} \sum_{i=1}^{N} w_{i} \Rightarrow I=q W \frac{\bar{\xi}}{\bar{w}}$

In addition, the sample variances and the sample covariance have to be determined:

$$
\begin{aligned}
s_{\xi}^{2} & =\frac{1}{N-1}\left(\sum_{i=1}^{N} \xi_{i}^{2}-N \bar{\xi}^{2}\right) \\
s_{w}^{2} & =\frac{1}{N-1}\left(\sum_{i=1}^{N} w_{i}^{2}-N \bar{w}^{2}\right) \\
s_{\xi w}^{2} & =\frac{1}{N-1}\left(\sum_{i=1}^{N} \xi_{i} w_{i}-N \bar{\xi} \bar{w}\right)
\end{aligned}
$$

Using these parameters, the variance of the random variable $\mu=\xi / w$ can be estimated as

$s_{\mu}^{2}=s_{\xi}^{2}-2 r s_{\xi w}^{2}+r^{2} s_{w}^{2}$,

where $r=\bar{\xi} / \bar{w}$ [11]. From $s_{\mu}$, the standard deviation of the current can be computed.

$s_{I}=q W \frac{s_{\mu}}{\sqrt{N} \bar{w}} \Rightarrow \frac{s_{I}}{I}=\frac{1}{\sqrt{N}} \frac{s_{\mu}}{\bar{\xi}}$

\section{Multi-band semiconductors}

The formalism described above assumes carrier transport in one band only. The extension of the formalism to many bands, however, is straightforward. The definitions of the normalization factors (32) and (33) have to be extended by summation over the band index $n$.

$Z(T)=\sum_{n} \int_{\text {BZ }} \mathrm{e}^{-\beta(T) \mathcal{E}_{n}(\mathbf{k})} \mathrm{d}^{3} k$

$V(T)=\sum_{n} \int_{\mathrm{BZ}}\left|v_{x}^{(n)}\left(\mathbf{k}_{0}\right)\right| \mathrm{e}^{-\beta(T) \mathcal{E}_{n}\left(\mathbf{k}_{0}\right)} \mathrm{d}^{3} k$

The band energy $\mathcal{E}_{n}$ denotes the energy of an electron with respect to the band edge energy $E_{C}$. Equation (35) defining the electron concentration at equilibrium has to account for a summation over the band index as well.

$n(\mathbf{r})=\frac{1}{4 \pi^{3}} \sum_{n} \int_{\text {BZ }} C(\mathbf{r}) \mathrm{e}^{-\beta(T) \mathcal{E}_{n}(\mathbf{k})} \mathrm{d}^{3} k$

The definition (36) of normalization factor $C$ remains unchanged.

The method of sampling an equilibrium trajectory yields random injection states of the form $\left(n_{0}, \mathbf{k}_{0}\right)$, where $n_{0}$ is the initial band index. 


\section{The combined backward-forward MC method}

In semiconductor devices, there are various processes which are caused by carriers with energies above a certain energy threshold. Such processes are impact ionization, carrier injection into the oxide, and the generation of interface traps due to hot carriers. To model such processes, only carriers with energies above the threshold need to be considered, whereas carriers with lower energies have no effect. Therefore, a good approximation in the modeling of such processes is to consider only those high energetic carriers that are able to surmount the energy barrier, and neglect the vast majority of carriers close to thermal equilibrium that get reflected on either side of the barrier.

This motivates the introduction of a combined backwardforward Monte Carlo method which simulates only those trajectories passing the energy barrier, see Fig. 1. In the first step of this method, a backward trajectory is started from the injection plane at $x_{0}$ with a random initial state $\left(\mathbf{k}_{0}, y_{0}\right)$. The contribution of this trajectory to the estimator (24) determines the statistical weight of this state:

$w\left(\mathbf{k}_{0}, \mathbf{r}_{0}\right)=f_{b}\left(\mathbf{k}_{b}, \mathbf{r}_{b}\right) \mathrm{e}^{\beta_{D}\left(H\left(\mathbf{k}_{b}, \mathbf{r}_{b}\right)-H\left(\mathbf{k}_{0}, \mathbf{r}_{0}\right)\right)}$

In the second step, a forward trajectory is started from the state $\left(-\mathbf{k}_{0}, y_{0}\right)$. This trajectory is assigned the statistical weight (77). The quantities of interest are computed as a weighted average over an ensemble of forward trajectories. Note that the backward trajectories are only needed to determine the weights. No averages are computed from those trajectories.

\section{Results and discussion}

The BMC method has been implemented in the full-band Monte Carlo simulator VMC [13]. Backward trajectories are constructed in the same manner as forward trajectories. Routines for the computation of the free flight and the afterscattering states can be used without modification.

As a test structure for the proposed simulation method, we use a planar n-channel MOSFET with a gate length of $L_{\mathrm{G}}=65 \mathrm{~nm}$, an effective oxide thickness of $t_{\mathrm{ox}}=2.5 \mathrm{~nm}$, and a channel width of $W=1 \mu \mathrm{m}$. Device geometry and doping profiles have been obtained by process simulation [14]. A sketch of the device structure is shown in Fig. 3. Room temperature is assumed for all simulations $\left(T_{\mathrm{D}}=300 \mathrm{~K}\right)$.

\subsection{Transfer characteristics}

The transfer characteristics have been calculated using the classical device simulator Minimos-NT [15,16], the conven-

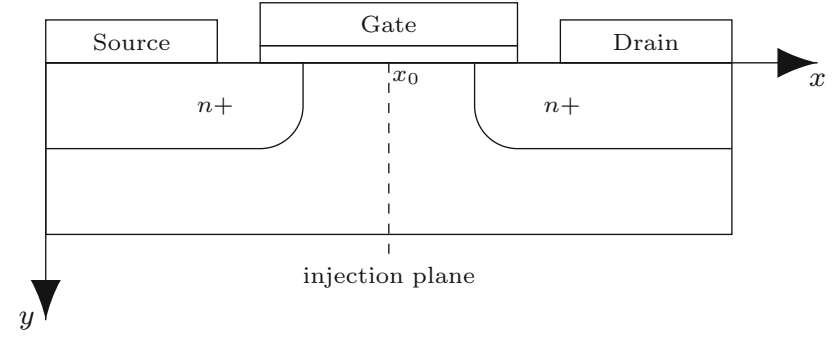

Fig. 3 Sketch of a MOSFET

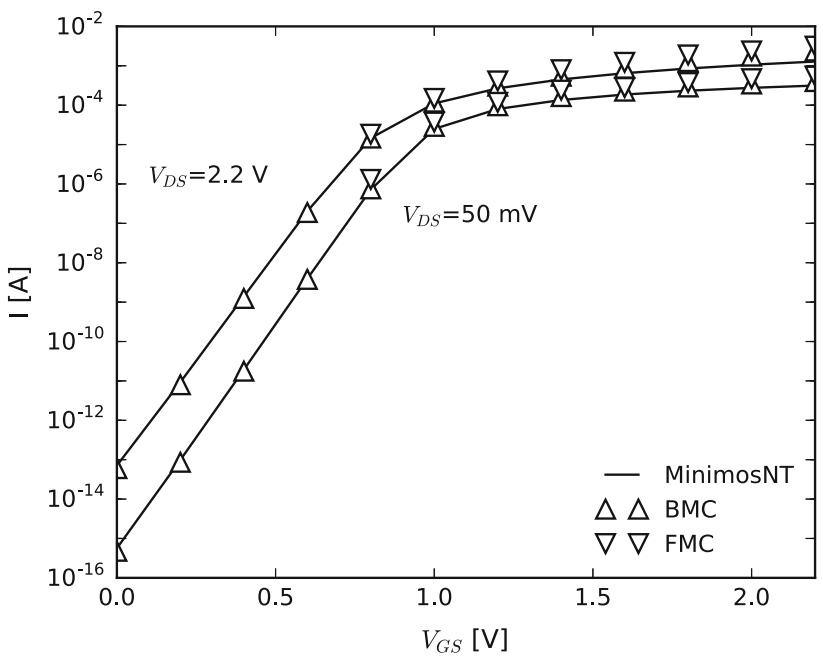

Fig. 4 Transfer characteristics of the $n$ MOSFET for two drain voltages, simulated with Minimos-NT, the backward and the forward MC methods

tional FMC method, and the novel BMC method. Each bias point is calculated with $10^{6}$ trajectories, both in the backward and forward methods. The maximum of the energy barrier determines the location of the injection plane. It is located at $x_{0}=10.2 \mathrm{~nm}$ relative to the left edge of the gate contact. Figure 4 shows good agreement between the classical device simulation and the MC simulations. The BMC method works well in the entire sub-threshold region, whereas the FMC method (without statistical enhancement) can cover only a few orders of magnitude of the current. The barrier height in the channel increases with decreasing gate voltage. Thus, at some point none of the forward trajectories will be able to surmount the barrier, giving an estimated current of $I=0$.

Further, the statistical error of the BMC method is depicted in Fig. 5. In a MOSFET the current component due to carriers injected at the source contact is nearly independent of the drain voltage, whereas the current component of carries originating from the drain contact depends strongly on the drain voltage. At $V_{\mathrm{DS}}=2.2 \mathrm{~V}$ the back diffusion current from the drain is extremely small, and the total current is dominated by forward diffusion, which will result in a low variance. At $V_{\mathrm{DS}}=50 \mathrm{mV}$, on the other hand, the back diffusion current is significant, and a stronger compensation of 


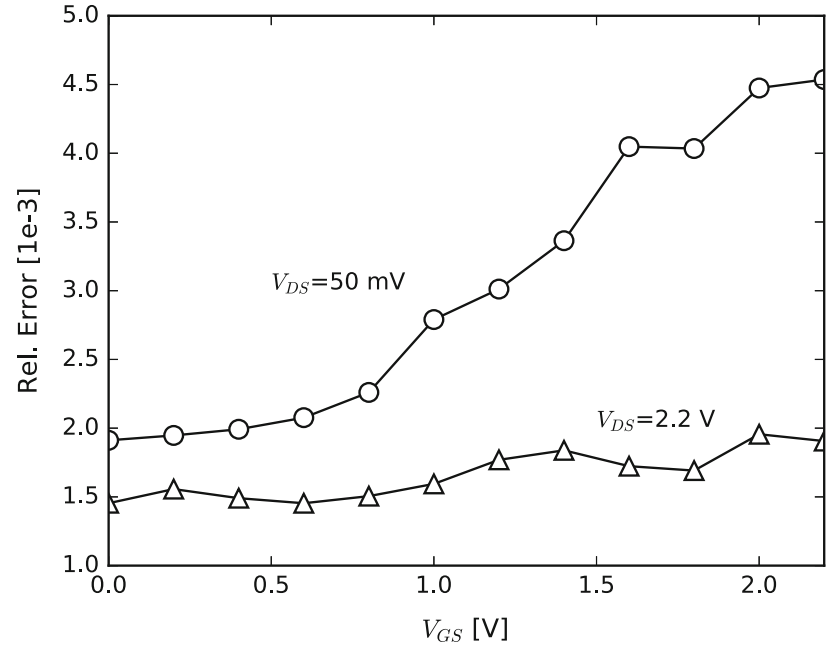

Fig. 5 Relative Error (relative standard deviation) of the current for two drain voltages. Each bias point is calculated with $10^{6}$ backward trajectories. The current estimator (40) was used

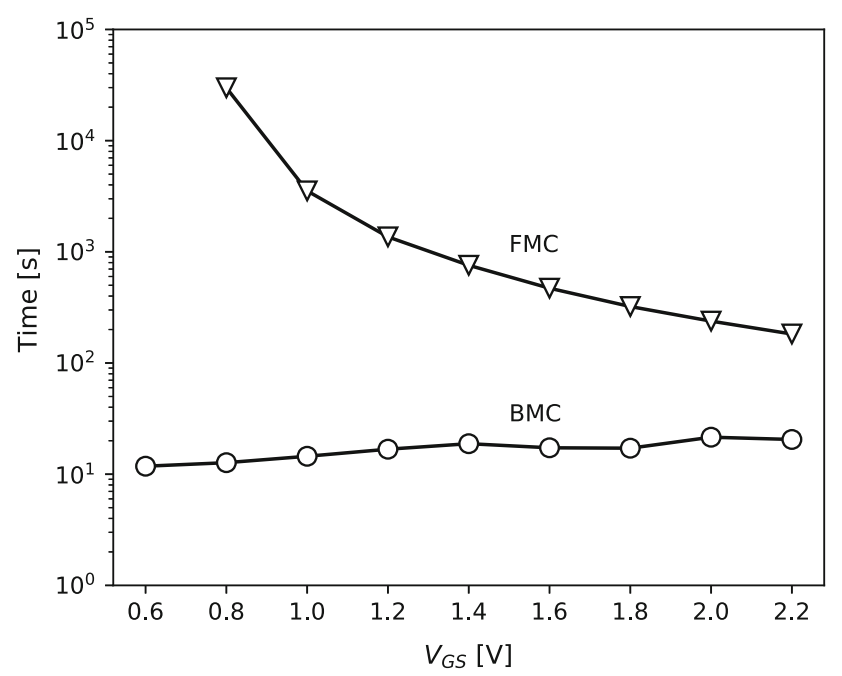

Fig. 6 Computation times by a single core of an Intel i7 processor. The operating points of the transfer characteristics at $V_{\mathrm{DS}}=2.2 \mathrm{~V}$ are considered. A relative standard deviation of $10^{-2}$ is assumed

the two current components takes place, which will result in a higher variance. This explanation, using the forward time picture also holds true in the backward time picture. There a vast difference in the two current components is reflected by a significant difference in the statistical weights of the forward and backward diffusing carriers.

In Fig. 6 we compare the computation times for a given error tolerance of $10^{-2}$. In the on-state $\left(V_{\mathrm{GS}}=2.2 \mathrm{~V}\right)$, $\mathrm{BMC}$ is about five times faster than FMC. Although in this operating point, the energy barrier in the channel is almost completely suppressed, many electrons injected at the source contact get reflected by the geometrical constriction at the source-channel junction. Since the BMC method need not simulate these reflected carriers, it shows a clear gain also in

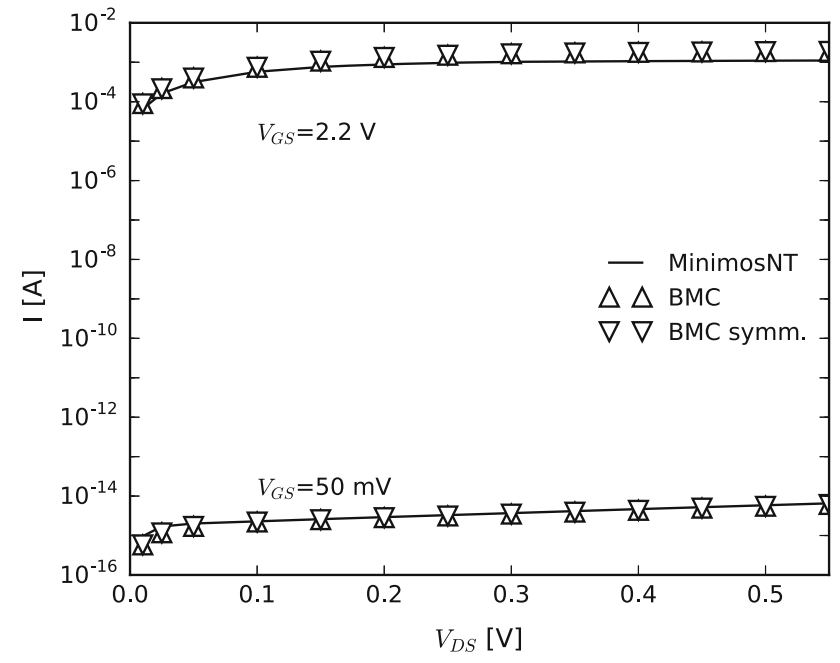

Fig. 7 Output characteristics of the MOSFET for two gate voltages, simulated with Minimos-NT, the forward and the backward MC methods

the on-state. The last point that could be simulated with FMC within a reasonable time was $V_{\mathrm{GS}}=0.8 \mathrm{~V}$. In this operating point, BMC is about 2300 times faster than FMC as shown in Fig. 6.

\subsection{Output characteristics}

Figure 7 compares the output characteristics computed by three different methods. As shown in Fig. 8, the statistical error decreases with increasing $V_{\mathrm{DS}}$, a trend already discussed in the previous section. The figure also shows that the variance of the symmetric estimator (63) is lower in the entire range of drain voltages. Especially at low $V_{\mathrm{DS}}$, where the device is approaching thermal equilibrium, the variance of the non-symmetric estimator tends to explode, whereas the variance of the symmetric estimator shows only a slight increase. In this regime, variance reduction by the symmetric estimator is particularly effective.

Evaluation of the symmetric estimator (63) requires the computation of two numerical trajectories. To obtain a fair comparison of the two estimators at equal computational cost, we compute $N=10^{6}$ realizations of the non-symmetric estimator (42) and only $N=5 \cdot 10^{5}$ realizations of the symmetric estimator. Despite the sample size being smaller in the latter case, this smaller sample gives the lower statistical error.

\subsection{Injection from a non-equilibrium distribution}

The injection distribution $f_{0}$ can be freely chosen and does not have any influence on the expectation value, but it does affect the estimator's variance. We demonstrate this fact by generating the random states $\mathbf{k}_{0}$ from a non-equilibrium 


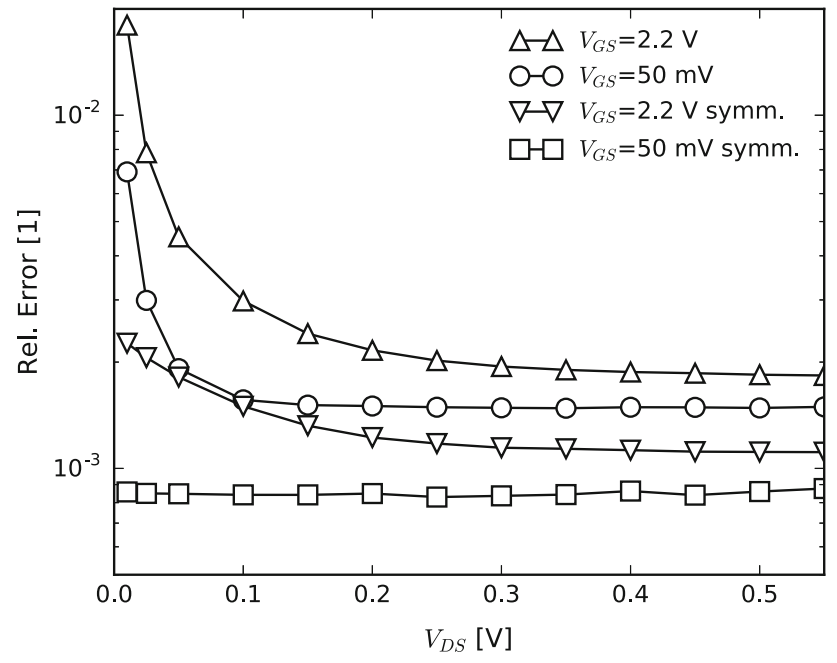

Fig. 8 Relative errors of the output characteristics at two gate voltages. The non-symmetric and the symmetric estimator based on the velocity-weighted Maxwellian are compared. Each bias point involves the calculation of $10^{6}$ backward trajectories

Maxwellian. The operating point is $V_{\mathrm{GS}}=0.6 \mathrm{~V}$ and $V_{\mathrm{DS}}=2.2 \mathrm{~V}$. The current is calculated using (46) in conjunction with the estimators (40) and (42). Figure 9 shows the independence of the estimated current from the injection temperature $T_{0}$.

The estimators' relative errors are compared in Fig. 10. Below $700 \mathrm{~K}$, estimator (42) shows less statistical error than estimator (40). For both estimators, the relative error shows a clear minimum, which can be explained as follows; the more the injection PDF $f_{0}$ resembles the real flux density $v_{x} f$, the lower is the current estimator's variance. From Fig. 10 we conclude that a velocity-weighted Maxwellian at $290 \mathrm{~K}$ is the best approximation of the real flux $v_{x} f$ at the injection plane. With increasing and decreasing $T_{0}$, the difference between $f_{0}$ and the real flux $v_{x} f$ becomes larger and the relative error increases. For $T_{0}>700 \mathrm{~K}$ the velocity-weighted Maxwellian is a worse approximation than the Maxwellian to the real flux $v_{x} f$ and thus shows a higher error.

\subsection{Energy distribution function}

Figure 11 shows the energy distribution function (EDF) at three surface points in the channel of the MOSFET. The forward MC simulation performed with $10^{9}$ trajectories can resolve only a few orders of magnitude of the EDF. Then again, with the backward MC method the EDF is calculated point-wise with $10^{4}$ trajectories per point using the estimator (24). The EDF shows a Maxwellian tail. One can compute as many orders of magnitude of the high-energy tail as needed.

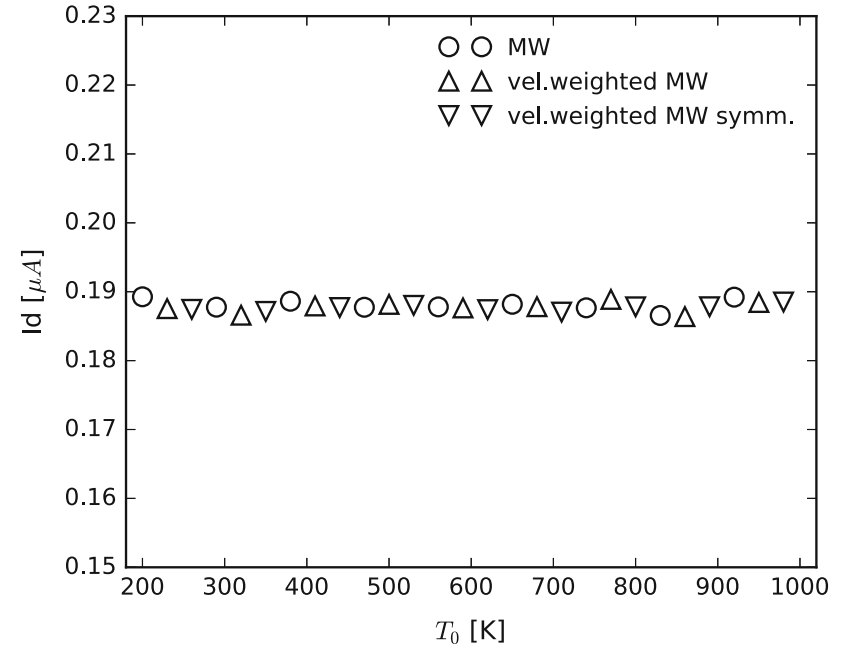

Fig. 9 Temperature stability of the current estimators (40), (42) and (63) which are based, respectively, on a Maxwellian (MW) and a velocityweighted Maxwellian (vel.weighted MW) injection PDF. Operating point is $V_{\mathrm{GS}}=0.6 \mathrm{~V}$ and $V_{\mathrm{DS}}=2.2 \mathrm{~V}$

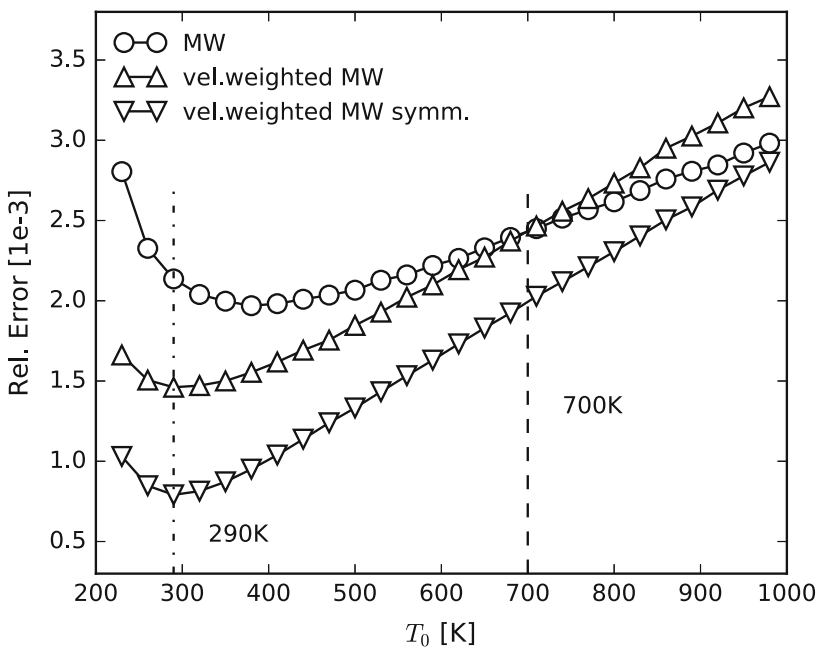

Fig. 10 Comparison of the relative errors of the current estimators (40), (42) and (63). Operating point is $V_{\mathrm{GS}}=0.6 \mathrm{~V}$ and $V_{\mathrm{DS}}=2.2 \mathrm{~V}$

\subsection{Hot carrier degradation}

In long channel devices and high-voltage MOSFETs degradation is triggered by hot carriers. It is assumed that degradation is caused by the breaking of $\mathrm{Si}-\mathrm{H}$ bonds at the silicon-oxynitride/silicon interface [14]. The bond dissociation rates are modeled by the acceleration integral, which has the general form [17].

$I_{A}=\sigma_{0} \int_{E_{\mathrm{th}}}^{\infty}\left(E-E_{\mathrm{th}}\right)^{p} v(E) f(E) g(E) \mathrm{d} E$, 


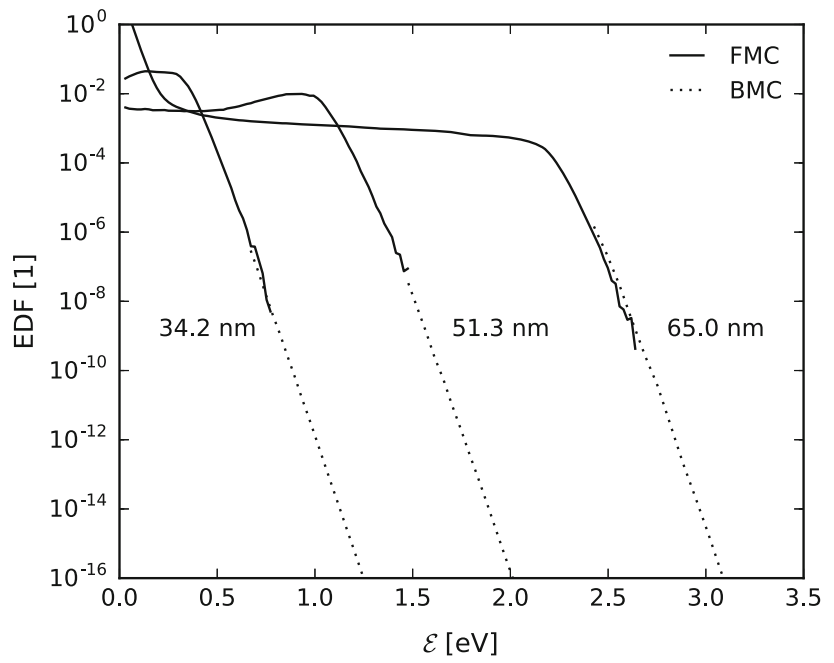

Fig. 11 Energy distribution function at three surface points in the channel. The distances from the left edge of the gate electrode are given. Operating point is $V_{\mathrm{GS}}=2.2 \mathrm{~V}$ and $V_{\mathrm{DS}}=2.2 \mathrm{~V}$. Solid lines: Forward MC simulation with $10^{9}$ trajectories. Dotted lines: High-energy tails computed with the backward MC method

where $E_{\mathrm{th}}$ denotes an energy threshold, $g(E)$ the density of states, and $v(E)$ the group velocity. For the purpose of MC estimation, we convert (78) into a k-space integral.

$I_{A}=\sigma_{0} \int_{\mathrm{BZ}} \Theta\left(E(\mathbf{k})-E_{\mathrm{th}}\right)\left(E(\mathbf{k})-E_{\mathrm{th}}\right)^{p}|\mathbf{v}(\mathbf{k})| f(\mathbf{k}) \mathrm{d}^{3} k$

Here $\Theta$ is the unit step function. For the process considered here in which one hot carrier is able to break a bond, an exponent of $p=11$ and an energy threshold of $E_{\mathrm{th}}=1.5 \mathrm{eV}$ are assumed [14].

We used the combined backward/forward MC method to evaluate the acceleration integral. The statistical average is calculated from the forward trajectories using the before-scattering method [18]. In this simulation, $10^{10}$ scattering events have been computed. To enhance the number of numerical trajectories at high energies the injection temperature $T_{0}$ has been raised significantly (5000 and $10,000 \mathrm{~K})$.

In Fig. 12 the MC results are compared to the result of ViennaSHE, a deterministic solver for the BE based on a spherical harmonics expansion of the distribution function [19]. Figure 12 shows that the MC results are independent from injection temperature. ViennaSHE predicts higher values in the first part of the channel where carrier heating is still moderate. We attribute this difference to the band structure model which is more approximate in ViennaSHE than it is in VMC.

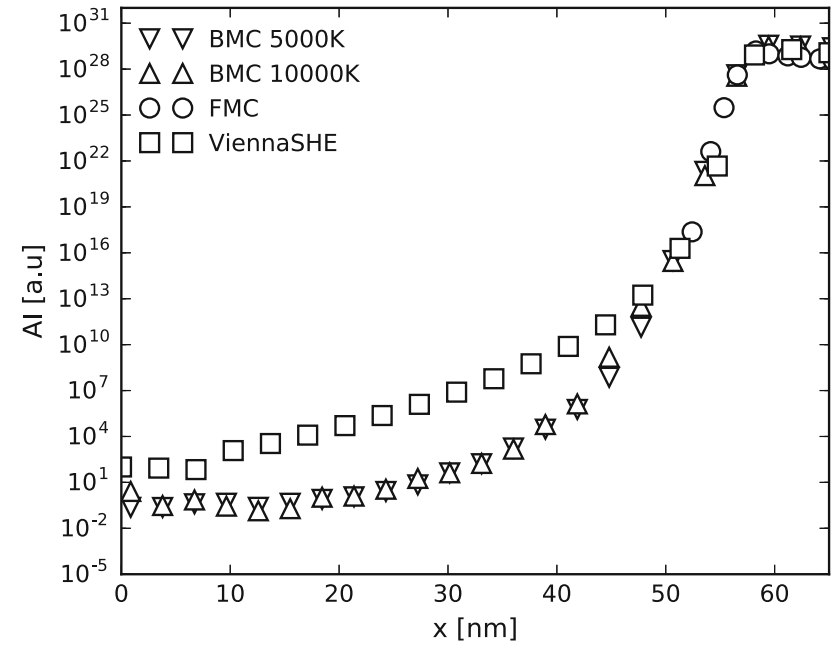

Fig. 12 Acceleration integral for a $65 \mathrm{~nm} n$ MOS, simulated with FMC and $\mathrm{BMC}$ method at different injection temperatures

\section{Conclusion}

A stable backward method has been implemented in a fullband Monte Carlo device simulator and used to study the electrical characteristics of an $n$-channel MOSFET. The method allows one to calculate the current in the entire subthreshold region including the leakage current in the off-state. Symmetric current estimators are proposed which produce less statistical error than the non-symmetric ones. This improvement is achieved for all operating conditions and is particularly large when thermal equilibrium is approached.

The current through a plane is calculated by Monte Carlo integration of the current density. For this integration, one has to assume a distribution of the sampling points which in the present case are the initial wave vectors of the backward trajectories. By assuming a Maxwellian distribution at elevated temperature the method will generate more sampling points at higher energies. This method of statistical enhancement reduces the statistical error of quantities that depend on the high-energy tail of the distribution function. It is shown that the estimated current is independent of the injection temperature, whereas the statistical error shows a clear minimum where the injection distribution most closely resembles the actual distribution. The proposed backward Monte Carlo method is able to estimate the energy distribution function in a chosen point in the $(r, k)$ phase space with desired accuracy. The high-energy tail of the distribution can be calculated point-wise. As an illustrative application we have estimated the so-called acceleration integral in the channel of a MOSFET and compared to values from the literature.

Acknowledgements Open access funding is provided by TU Wien (TUW). The financial support of the the Austrian Research Promotion Agency (FFG), project MORAFLASH (contract No. 850660) is thank- 
fully acknowledged. The computational results presented have been achieved in part using the Vienna Scientific Cluster (VSC).

Open Access This article is distributed under the terms of the Creative Commons Attribution 4.0 International License (http://creativecomm ons.org/licenses/by/4.0/), which permits unrestricted use, distribution, and reproduction in any medium, provided you give appropriate credit to the original author(s) and the source, provide a link to the Creative Commons license, and indicate if changes were made.

\section{A Generation of the injection states}

A single-particle MC simulation is performed with the motion of the particle confined to a box of constant length, as shown in Fig. 13. An equilibrium distribution is obtained by setting the electric field to zero. Every time the particle hits a boundary and gets reflected, its current state is used as an initial state of for the BMC method. The distribution of the $\mathbf{k}$-values generated with this scheme is a velocity-weighted Maxwellian [20].

$$
f_{0}(\mathbf{k})=\left|v_{\perp}(\mathbf{k})\right| f_{M}(\mathbf{k})
$$

Here $\left|v_{\perp}(\mathbf{k})\right|$ is the velocity component normal to the boundary. The width of the Maxwellian is determined by the temperature one uses for the calculation of the phonon scattering rates.

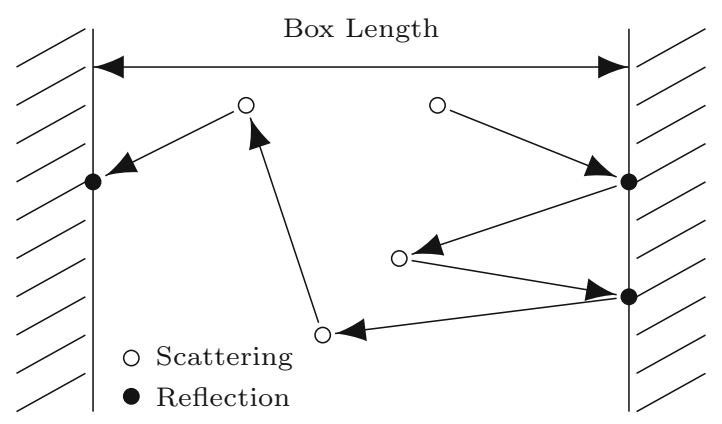

Fig. 13 Box method sampling

\section{References}

1. Jacoboni, C., Lugli, P.: The Monte Carlo Method for Semiconductor Device Simulation. Springer, New York (1989)

2. Jungemann, C., Meinerzhagen, B.: Hierarchical Device Simulation. Springer, New York (2003)

3. Jacoboni, C., Poli, P., Rota, L.: A new Monte Carlo technique for the solution of the Boltzmann transport equation. Solid-State Electron 31(3/4), 523 (1988)

4. Nedjalkov, M., Vitanov, P.: Iteration approach for solving the Boltzmann equation with the Monte Carlo method. Solid-State Electron 32(10), 893 (1988)
5. Kosina, H., Nedjalkov, M., Selberherr, S.: In: Lecture Notes in Computer Science, vol. 2907, pp. 170-177. Springer, New York, (2003)

6. Kampl, M.: Implementation of a backward Monte Carlo algorithm to investigate hot carriers in semiconductor devices. In: Master's Thesis, Technische Universitt Wien, Vienna, (2015)

7. Lugli, P., Ferry, D.: Degeneracy in the ensemble Monte Carlo method for high-field transport in semiconductors. IEEE Trans. Electron Dev. ED-32(11), 2431 (1985)

8. Kosina, H., Nedjalkov, M., Selberherr, S.: Theory of the Monte Carlo method for semiconductor device simulation. IEEE Trans. Electron Dev. 47(10), 1898 (2000)

9. Chambers, R.: The kinetic formulation of conduction problems. Proc. Phys. Soc. (Lond.) A65, 458 (1952)

10. Kosina, H., Nedjalkov, M., Selberherr, S.: The stationary Monte Carlo method for device simulation. Part I. Theory. J. Appl. Phys. 93(6), 3553 (2003)

11. Rubinstein, R.Y.: Simulation and the Monte Carlo Method. Wiley, Hoboken (1981)

12. Newmann, M.E.J., Barkema, G.T.: Monte Carlo Methods in Statistical Physics. Oxford University Press, Oxford (1999)

13. Kampl, M., Kosina, H.: In: Conference Proceedings of International Workshop on Computational Nanotechnology, pp. 147-148 (2017)

14. Tyaginov, S.E., Bina, M., Franco, J., Osintsev, D., Triebl, O., Kaczer, B., Grasser, T.: In: Conference Proceedings of International Reliability Physics Symposium, pp. XT16.1-XT16.8 (2014)

15. Minimos-NT User Manual (2018). www.globaltcad.com. Accessed 3 Apr 2018

16. Simlinger, T., Brech, H., Grave, T., Selberherr, S.: Simulation of submicron double-heterojunction high electron mobility transistors with MINIMOS-NT. IEEE Trans. Electron Dev. 44, 700 (1997)

17. McMahon, W., Matsuda, K., Lee, J., Hess, K., Lyding, J.: In: Proceedings of International Conference on Modelling and Simulation, vol. 1. pp. 576-579. (2002)

18. Jacoboni, C., Reggiani, L.: The Monte Carlo method for the solution of charge transport in semiconductors with applications to covalent materials. Rev. Mod. Phys. 55(3), 645 (1983)

19. ViennaSHE User Manual (2018). http://viennashe.sourceforge. net/doc/. Accessed 3 Apr 2018

20. Lundstrom, M.: Fundamentals of carrier transport. Cambridge University Press, Cambridge (2000) 